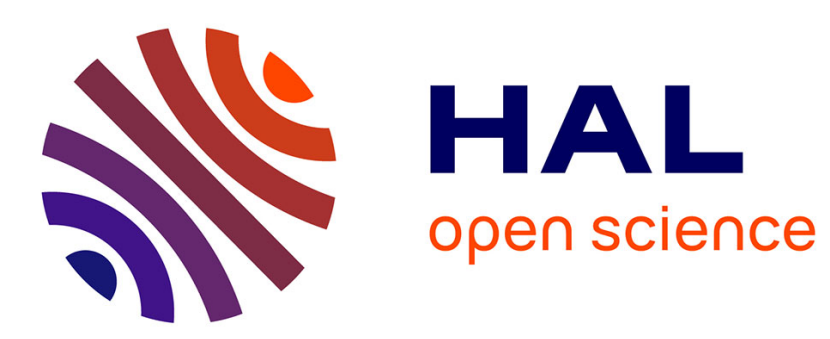

\title{
Direct Numerical Simulation and Wall-Resolved Large Eddy Simulation in Nuclear Thermal Hydraulics
}

Iztok Tiselj, Cédric Flageul, Jure Oder

\section{To cite this version:}

Iztok Tiselj, Cédric Flageul, Jure Oder. Direct Numerical Simulation and Wall-Resolved Large Eddy Simulation in Nuclear Thermal Hydraulics. Nuclear Technology, 2019, pp.1-15. 10.1080/00295450.2019.1614381. hal-02154872

\section{HAL Id: hal-02154872 \\ https://hal.science/hal-02154872}

Submitted on 5 Jul 2019

HAL is a multi-disciplinary open access archive for the deposit and dissemination of scientific research documents, whether they are published or not. The documents may come from teaching and research institutions in France or abroad, or from public or private research centers.
L'archive ouverte pluridisciplinaire HAL, est destinée au dépôt et à la diffusion de documents scientifiques de niveau recherche, publiés ou non, émanant des établissements d'enseignement et de recherche français ou étrangers, des laboratoires publics ou privés. 


\title{
Direct Numerical Simulation and Wall-Resolved Large Eddy Simulation in Nuclear Thermal Hydraulics
}

\author{
Iztok Tiselj, ${ }^{*}$ Cedric Flageul, and Jure Oder \\ Jožef Stefan Institute, Jamova 39, 1000 Ljubljana, Slovenia
}

Received March 15, 2019

Accepted for Publication April 30, 2019

\begin{abstract}
The critical review discusses the most accurate methods for description of turbulent flows: the computationally very expensive direct numerical simulation (DNS) and slightly less accurate and slightly less expensive large eddy simulation (LES) methods. Both methods have found their way into nuclear thermal hydraulics as tools for studies of the fundamental mechanisms of turbulence and turbulent heat transfer. In the first section of this critical review, both methods are briefly introduced in parallel with the basic properties of the turbulent flows. The focus is on the DNS method, the so-called quasi-DNS approach, and the coarsest turbulence modeling approach discussed in this work, which is still on the very small-scale, wall-resolved LES. Other, coarser turbulence modeling approaches (such as wall-modeled LES, Reynolds Averaged Navier-Stokes (RANS)/LES hybrids, or RANS) are beyond the scope of the present work. Section II answers the question: "How do the DNS and LES methods work?" A short discussion of the computational requirements, numerical approaches, and computational tools is included. Section III is about the interpretation of the DNS and LES results and statistical uncertainties. Sections IV and V give some examples of the DNS and wall-resolved LES results relevant for nuclear thermal hydraulics. The last section lists the conclusions and some of the challenges that might be tackled with the most accurate techniques like DNS and LES.
\end{abstract}

Keywords - Direct numerical simulation, wall-resolved large eddy simulation.

Note - Some figures may be in color only in the electronic version.

\section{INTRODUCTION}

Direct numerical simulations (DNSs) and wallresolved large eddy simulations (LESs), often denoted with common-name high-fidelity simulations, represent an important research tool in fluid dynamics and heat and mass transfer. These tools complement the experimental work and industrial computational fluid dynamics (CFD) methods based on Reynolds Averaged Navier-Stokes (RANS) models of turbulence. Data obtained with carefully performed high-fidelity simulations can have the same status as accurate measurements. These data are relevant for certain rather simple geometries at low and moderate Reynolds numbers and can elucidate the details of the flow structures that cannot be reproduced with less

*E-mail: iztok.tiselj@ijs.si accurate RANS models, especially for flows with strong separations of the boundary layers and reattachments. Two examples of such flows presented in this critical review are flow over backward-facing step (BFS) and the flow through the pebble bed reactor. And while the BFS flow can be accurately assessed with experiments, pebble beds are very challenging for experimental observations. In such cases, high-fidelity methods are thus the best option to obtain accurately resolved data without the introduction of significant modeling uncertainties or experimental distortions.

The computational costs of these methods prevent their use for many high Reynolds industrial flows, especially those in complex geometries. However, even for such flows, where RANS models remain the only reasonable option for simulations, the high-fidelity methods can contribute to the development of the turbulence models that are the key ingredient of the RANS models. 


\section{I.A. What Is Turbulence? What Is DNS?}

This critical review discusses very accurate numerical simulations of turbulent flows. Thus, we can use a rather nonstandard definition of turbulence: "numerical" turbulence that emerges as a solution of the NavierStokes equations, where

$$
\nabla \cdot \vec{u}=0
$$

and

$$
\frac{\partial \vec{u}}{\partial t}=-\nabla \cdot(\vec{u} \vec{u})+v \nabla^{2} \vec{u}-\frac{\nabla p}{\rho}
$$

is equivalent to the "measurable" properties of the corresponding fluid flow. The nonlinear Eqs. (1) and (2) are written for the constant fluid properties flow, where $\vec{u}$ denotes flow velocity, $v$ denotes kinematic viscosity, $p$ denotes pressure, and $\rho$ denotes the density of the fluid. Equation (2) can be rewritten in dimensionless form as

$$
\frac{\partial \vec{u}^{*}}{\partial t}=-\nabla \cdot\left(\vec{u}^{*} \vec{u}^{*}\right)+\frac{1}{\operatorname{Re}} \nabla^{2} \vec{u}^{*}-\nabla p^{*}
$$

where the Reynolds number is defined with characteristic dimension of the flow geometry $L$ (pipe diameter, for example) and characteristic velocity $U$ (mean velocity in the pipe): $R e=U L / v$.

It is rather well known and widely accepted ${ }^{1}$ that unsteady solutions of these equations obtained at an appropriate Reynolds number and with suitable numerical approaches show excellent agreement with measurements in turbulent incompressible Newtonian fluids. This type of numerical simulation is denoted as the DNS. The feasibility of DNS is a consequence of the fact that the smallest scales of the turbulent flow are not infinitely small. They are finite and their dimension represents the key information for DNSs of turbulent flows: A DNS is a simulation with sufficient spatial and temporal resolution to capture the smallest scales of the turbulent flow.

The "agreement" of experiments and DNSs does not mean the same temporal development of the dependent variables in a selected point of computational domain and in the equivalent point of the experimental device. As solutions of the nonlinear Eqs. (1) and (2) are chaotic, the agreement means that the same statistical behavior of the numerical solution and measured signal can be observed. And like in any other nonlinear system, any minor change in initial or boundary conditions, numerical method, grid, time step, etc., can result in a different instantaneous solution of the turbulent field after sufficiently long time. However, as long as we do not compromise the accuracy of numerical simulations, their statistical properties remain unchanged.

\section{I.B. Length and Timescales of the Turbulent Flows}

A few more words about the characteristic scales is needed before we define the LES. The smallest length and timescales of turbulence, which can be roughly interpreted as a dimension and an inverse of angular velocity of the smallest turbulent vortices in the flow, were given by Kolmogorov (see the textbook of $\mathrm{Pope}^{1}$ for background):

$$
\eta=\left(v^{3} / \varepsilon\right)^{\frac{1}{4}}
$$

and

$$
\tau=\eta^{2} / v
$$

where $\varepsilon$ represents the average rate of dissipation of turbulence kinetic energy per unit mass. Equation (4) is useful for a posteriori evaluation of the turbulence scales, while the rough estimates that relate the smallest and the integral scales can be used a priori:

$$
\eta=L \operatorname{Re}_{T}^{-\frac{3}{4}}
$$

and

$$
\tau=t_{0} \operatorname{Re}_{T}^{-\frac{1}{2}}
$$

The integral scales $L$ and $u_{0}=L / t_{0}$ are often the quantities used to evaluate the Reynolds number, while the turbulent Reynolds $\mathrm{Re}_{T}$ number is not evaluated with the bulk velocity but with the square root of the turbulent kinetic energy (typically $10 \%$ to $20 \%$ of the bulk velocity). An important consequence of Eq. (5) for the DNSs, where the domain of length $L$ must be discretized roughly into $n$ intervals of length $\Delta x=\eta$, is $n \sim \operatorname{Re}_{T}^{3 / 4}$ and in threedimensional (3-D) $n^{3} \sim \operatorname{Re}_{T}{ }^{9 / 4}$. Numerical experiments show that the fully resolved DNS is obtained at $\Delta x \approx 2 \eta$.

Thus, it would take $10^{12}$ cells to capture the flow in a single channel of the pressurized water reactor (PWR) fuel element at $\operatorname{Re}_{T}=10^{5}\left(\operatorname{Re}=10^{6}\right)$ with DNS precision. The largest meshes in the fluid dynamics simulations running on the fastest supercomputers today contain 
$\sim 10^{10}$ to $10^{11}$ cells. $^{2}$ It is thus clear that computationally extremely expensive DNS cannot be foreseen as a tool for industrial applications in the near future and that there are strong incentives to develop cheaper methods and models for simulations of turbulent flows.

Although computationally expensive, DNS results are useful as they help us to understand the fundamental physics of the turbulence. They are useful also for improving and developing the LES and RANS models of turbulence.

\section{I.C. Quasi-DNS}

What happens when a DNS computer code is running on a mesh that is too coarse to capture all the smallest scales? Turbulence books ${ }^{1}$ suggest that the smallest scales convert the kinetic energy of the turbulent fluctuation into internal energy. If a minor part of these scales are missing in the simulation and remain unresolved, the remaining resolved scales can take over their task. Such simulations are denoted as quasi-DNS or underresolved DNS. They are typically using resolution that is $\sim 2$ to 5 times too coarse to capture the smallest relevant scales, and their results are still very close to the measured ones. According to today's standards, ${ }^{3}$ even some of the first DNS studies ${ }^{4}$ can be categorized as a quasi-DNS rather than a true DNS. When the quasi-DNS resolution is 2 to 3 times coarser than in DNS, the tiny differences are usually seen only in the nonphysical behavior of the spectra in the range of the smallest turbulent fluctuations. If the quasi-DNS resolution is 4 to 5 times coarser, the differences are seen also in the profiles of the velocity fluctuations (or fluctuations of other quantities). For further coarsening of the mesh $(\Delta x \approx 20 \eta \ldots)$, numerical results show larger and larger discrepancies from the measurements. Depending on the type of the numerical scheme, the DNS code used in quasi-DNS mode will typically "explode" due to insufficient dissipation of the short wavelength fluctuations (numerical instability) when very coarse meshes are used.

\section{I.D. Large Eddy Simulation}

In order to accurately simulate turbulent flows with resolutions that are roughly 10 times coarser in each direction than in DNS, additional (semi)empirical models (subgrid-scale models) are needed to dissipate the turbulent kinetic energy of the smallest turbulent scales that are not explicitly captured in the simulation. Such models are known as LES (Ref. 1). The LES approach can be very accurate due to the isotropic behavior of the smallest eddies. This property allows rather simple subgrid-scale models on up to $10^{3}$ times coarser meshes in comparison with DNS. However, an accurate LES approach where the near-wall layers are accurately resolved (wallresolved LES) must use near-wall resolution that is only marginally coarser than in DNS, and the typical meshes in wall-resolved LES are in most cases between one and two orders of magnitude coarser than in DNS.

The LES approach is extremely valuable for the investigation of certain high Reynolds number flows and for the development and assessment of new RANS turbulence models. The LES methods are also useful for the prediction of complex and unsteady flows where performance of the other turbulence models is not adequate. The DNS and wall-resolved LES can be fully reproducible and will be more and more important for verification and validation and uncertainty quantification in (reliable) CFD.

\section{I.E. Brief History of Nuclear-Related DNS and LES}

Instead of a detailed history of DNS and LES computations, we mention only some of the review papers and textbooks that are relevant for the field. The first decades of DNS simulations are described in a 1998 paper by Moin and Mahesh ${ }^{5}$ and in the book by Pope. ${ }^{1}$ The story starts in the 1970s with the first simulations of homogeneous turbulence using spectral schemes. These were followed by DNS studies that examined the effects of shear, irrotational strain, and rotation on the homogeneous turbulence in the 1980s. The first wall-bounded DNS was performed in 1987 (Ref. 4), roughly at the same time the first boundary layer simulations were performed. The first heat (mass) transfer studies, which are of particular importance also for nuclear thermal hydraulics, were performed in the early 1990s (Ref. 6). The first flows of moderate complexity (riblets, back step, boundary layer separation) and the first compressible flows were also analyzed with DNS in the late 1990s. Our first contributions to the field are from 2001 when we performed DNS analyses of the turbulent conjugate heat transfer. $^{7,8}$ The DNS in this millennia has spread to all fields of engineering. Although it is most commonly used in simple geometries, ${ }^{9}$ it is not limited to such and today's simulations are performed in different geometries. Among the recent examples is flow in the BFS geometry with natural convection, ${ }^{10}$ and a complex DNS of pebble bed reactor by Fick et al. ${ }^{11}$ The largest DNS runs performed in the past years are by Lee and Moser: ${ }^{12}$ channel flow DNS of at friction Reynolds number around 5200 with approximately $1.2 \times 10^{11}$ points. Similar 
investigations of the near-wall region were performed by Yamamoto and Tsuji ${ }^{13}$ at friction Reynolds number 8000 with a DNS on approximately $2.2 \times 10^{11}$ points.

The history of LES, as a computationally less demanding approach, is even richer and is reviewed in Refs. 1, 14, and 15. The first works of Smagorinsky and Lilly in meteorology and Deardorff in engineering go back to the 1960s and 1970s, respectively. Since then, the popularity of LES grew quickly in various fields of natural and engineering sciences, including nuclear technology. Considering only single-phase flows relevant for nuclear thermal hydraulics, most of them are related to turbulent mixing problems, including temperature mixing or the mixing of chemical components in a multicomponent mixture (boron in water, hydrogen in air, etc.) with possible effects of density gradients and natural circulation. Some of these are addressed in Sec. V. Possible applications of LES consider the mixing of cold water coming from the broken loop and hot water coming from the others in the reactor pressure vessel (RPV) downcomer during the steam line break accident, pressurized thermal shock in the RPV downcomer, stratification and hot-leg temperature heterogeneities, thermal fatigue, erosion, corrosion, and deposition. Heterogeneous flow distribution [e.g., in the steam generator (SG) inlet plenum causing vibrations, etc.], the boiling water reactor lower plenum flow, hydrogen distribution in containment, and chemical reactions/ combustion/detonation are also candidates for LES analyses. Several specific flows relevant for advanced reactors (gas cooled, pebble bed, liquid metal cooling) can also be analyzed with LES.

Among the mixing problems listed above only thermal fatigue requires explicit accurate prediction of unsteady temperature field. That almost excludes RANS approaches and gives a strong added value to the LES.

\section{HOW DOES IT WORK?}

\section{II.A. Spectral Numerical Schemes}

The key prerequisite for successful DNS and LES analyses are accurate numerical schemes that can be successfully parallelized and implemented on today's parallel computers. The first DNS and LES studies in simple geometry of an infinite channel were performed with spectral schemes where solutions of the NavierStokes [Eqs. (1) and (2)] are written as a finite Fourier series and/or series of some other basis functions like Chebyshev polynomials. ${ }^{1,4}$ Fourier transformations are used to convert the solutions into physical space, where the nonlinear terms are evaluated (pseudo-spectral scheme). The aliasing error that stems from the forward and backward Fourier transformations can be removed by computing the nonlinear terms on a number of modes 1.5 times larger in each direction ${ }^{4}$ or with some other, more efficient technique. ${ }^{16}$

Spectral schemes are still recognized as the most accurate techniques for DNS studies; however, their parallelization on massively parallel computers is moderately efficient, and they are limited to rather simple geometries (cuboid, cylinder) and boundary conditions. Authors of the first turbulent channel DNS studies, which were performed 30 years ago, are still maintaining and upgrading their databases with open access data. ${ }^{1,17}$ A huge DNS database in open access domain is maintained also by Johns Hopkins University. ${ }^{18}$ The DNS heat transfer databases based on spectral scheme results can be found in Refs. 6 and 7. Conjugate heat transfer results for moderate and low Prandtl number fluids can be found in Refs. 8 and 19, respectively.

Dimensional variables in near-wall flows, which are mainly relevant for nuclear applications, are often converted in dimensionless form by using wall units. The reference length scales and velocities depend on the wall friction and viscosity. ${ }^{1}$ The length in wall units is defined with the friction velocity $u_{\tau}$ as

$$
y^{+}=\frac{u_{\tau} y}{v}
$$

and

$$
u_{\tau}=\sqrt{v \frac{\partial U}{\partial y}}
$$

where $y$ represents wall-normal distance and $U$ represents mean velocity. Typical resolution (i.e., the distance between the collocation points of the spectral scheme) in DNS studies of turbulent channel flows at friction Reynolds numbers $\mathrm{Re}_{\tau}=u_{\tau} h / v$ ( $h$ is channel half-width) between 180 and 2000 (Refs. 17 and 20) is $\Delta x^{+}=10$ to $18, \Delta z^{+}=5, \Delta y^{+}<1$ (near the wall), and $\Delta y^{+}=8$ (channel center), in the streamwise, spanwise, and wall-normal directions, respectively. This resolution guarantees the accuracy of turbulent statistics for most of the relevant quantities. Nevertheless, a detailed resolution study performed by Vreman and Kuerten ${ }^{3}$ has shown that some higher-order statistics of turbulent flow (e.g., turbulent dissipation) require a finer resolution, namely $\Delta x^{+}=6$ and $\Delta z^{+}=4$, in the streamwise and 
spanwise directions, while in the wall-normal direction they recommend $\Delta y^{+}=3$ in the center for the channel and $\Delta y^{+}=1$ in the near-wall region at $y^{+} \sim 12$. The resolution requirements proposed by Vreman and Kuerten are actually close to the theoretical prediction from Eq. (4), for which $\Delta x, \Delta y, \Delta z \approx 2 \eta$ (Kolmogorov scale is around two wall units near the wall and approximately four wall units in the center). Strict classification would probably put the earlier DNS studies, ${ }^{4,7,17}$ including our heat transfer DNS (Refs. 7 and 8) into the category of quasi-DNS simulations. Typical resolutions of various DNS/LES channel flow studies performed with different numerical schemes, including spectral, are collected in Table I.

The evaluation of wall units in more complex geometries is not exact as in the channel flow, but relies on correlations of friction coefficient (empirical or semiempirical). These correlations are more reliable for simple geometries and less accurate for complicated engineering geometries, e.g., PWR subchannel. A safer DNS mesh design approach is iterative: test run is performed, turbulent kinetic energy and its dissipation calculated, and the Kolmogorov scale that follows from $k(\varepsilon)$ is compared with the grid size. The loop continues until the grid size is comparable to the Kolmogorov scale.

Temporal discretization of the equations in spectral schemes is less accurate than the spatial one: secondorder accurate finite difference schemes in the first codes were later upgraded into third- and fourth-order schemes (typically Runge-Kutta). For the choice of the time-step size in DNS, the physical constraints and numerical accuracy and stability constraints must be met. The time step should not exceed a fraction of the Kolmogorov timescale to keep DNS accuracy. However, numerical stability and numerical accuracy criteria are typically more stringent. For example, in our DNS (Ref. 7) with second-order accurate time scheme, a timestep size is chosen such that the Courant number is around 0.1 to ensure sufficient numerical accuracy, although the scheme is stable for Courant numbers up to 0.5 .

\section{II.B. Filtering of LES Equations}

Mathematical models of LES are more complex than in DNS because the kinetic energy sink in the high wave number fluctuations is not simulated and must be taken into account with empirical models. An ideal LES should consider only the motions on the length scales larger than the filter width $\Delta_{\text {filter }}$, while the actual resolution of the numerical scheme should be more precise: $\Delta x<\Delta_{\text {filter }}$ so that the artificial dissipation of the smallest numerically resolved scales in the simulation occurs roughly on the interval $\left[\Delta x, \Delta_{\text {filter }}\right]$. Nevertheless, the recommendation that grid resolution in LES should be $\Delta_{\text {filter }} / 4<\Delta x<\Delta_{\text {filter }} / 2$ in order to reduce numerical errors, is almost never followed $^{21}$ due to the high computational costs. Grid resolution $\Delta x=\Delta_{\text {filter }}$ is used in most of today's LESs.

The first consequence of LES studies that are done with the filter width equal to grid resolution is strong

TABLE I

Comparison of Typical Grid Resolutions and Time Steps of Various DNS/LES Channel Flow Studies

\begin{tabular}{|c|c|c|c|c|c|c|}
\hline $\begin{array}{c}\text { Approach } \\
\text { (Computational Domain } \\
4 \pi \times 2 \times 4 \pi / 3)\end{array}$ & $\Delta x^{+}$ & $\Delta y_{\text {min }}^{+}$ & $\Delta y_{\text {max }}^{+}$ & $\Delta z^{+}$ & $\begin{array}{c}\quad \Delta t \\
\text { for Second- } \\
\text { Order Time } \\
\text { Scheme }\end{array}$ & $\begin{array}{c}\text { Relative } \\
\text { Computational } \\
\text { Effort }\end{array}$ \\
\hline & Streamwise & \multicolumn{2}{|c|}{ Wall Normal } & Spanwise & $\begin{array}{r}\text { Normalized } \\
\text { with } h / u_{\tau}\end{array}$ & $\begin{array}{l}\text { (Order of } \\
\text { Magnitude) }\end{array}$ \\
\hline $\begin{array}{l}\text { Fully resolved spectrum DNS } \\
\left.\text { (Vreman and Kuerten }^{3}\right)\end{array}$ & 5.9 & 0.01 & 2.9 & 3.9 & 0.00025 & 1 \\
\hline $\begin{array}{l}\text { Spectral DNS (Tiselj and } \\
\text { Cizelj }^{19} \text { ) }\end{array}$ & 17.7 & 0.01 & 4.4 & 5.9 & 0.00015 & $10^{-1}$ \\
\hline $\begin{array}{l}\text { Spectral elements DNS } \\
\left(\text { Nek5000, Oder et al. }{ }^{34}\right)\end{array}$ & 12.6 & 1 & 2.8 & 4.2 & 0.0002 & $10^{-1}$ \\
\hline $\begin{array}{l}\text { Finite difference DNS } \\
\quad(\text { Vreman and Kuerten } \\
\text { ) }\end{array}$ & 8.8 & 0.98 & 4.4 & 5.9 & 0.0010 & $10^{-1}$ \\
\hline $\begin{array}{l}\text { Finite volume LES } \\
\left(\text { Code_Saturne }{ }^{49}\right)\end{array}$ & 30 & 1 & 15 & 15 & 0.00016 & $10^{-2}$ to $10^{-3}$ \\
\hline
\end{tabular}


coupling of the physical LES subgrid-scale model and the numerical errors. The LES subgrid-scale models stem from the tedious formal filtering procedures performed on the basic Navier-Stokes equations, which are discussed in several textbooks and papers. ${ }^{1,15}$ The overview of the so-called implicit LES, where numerical dissipation is carefully (or less carefully) used to ensure dissipation of the smallest turbulent scales, can be found in Ref. 22. Although the theoretical background of the implicit LES is not entirely clarified and the approach remains controversial, many authors recognize it as a viable option for LES simulations and also for quasiDNS computations. ${ }^{21,23}$

The second consequence of the rather blurred interface between the physical and numerical dissipation of the smallest scales in LES studies is relevant for the present work, as it allows us to skip the rather complex mathematics behind the derivation of LES equations and subgrid-scale models. Thus, instead of a precise mathematical definition we give a rather vague description of LES subgrid-scale models, which are considerably different in various numerical schemes. For the spectral schemes, LES filtering is rather simple-the subgrid-scale models are introduced as an additional viscosity, which is a function of the wave number and additional viscosity for lower wave numbers (largescale vortices) is zero, while the subgrid viscosity is nonzero in the range of the smallest eddies that are still resolved by the simulation.

\section{II.C. Finite Difference and Finite Volume Schemes}

Various finite volume and finite difference schemes are today used for DNS and are probably the most popular approaches for LES studies. The reason why they are preferred to spectral schemes is easier implementation in complex geometries and also simpler implementation of various boundary conditions. The finite difference methods are thoroughly documented in several textbooks. ${ }^{24,25}$ A special class of finite difference schemes - compact schemes, which are high-order spatial schemes that increase accuracy and resolution without widening the computational stencil-are popular in DNS. Compact finite difference schemes have been used for DNSs since the 1990s. More recently, these schemes were implemented in Incompact3d, one of the most important open source DNS codes. ${ }^{26}$

In a finite volume scheme mass, momentum, and energy balance equations are expressed in their integral form over each of the nonoverlapping control volumes that form the computational domain. The applicability of finite volume schemes on unstructured grids in complex geometries is one of the reasons why most commercial codes are based on this approach. The finite volume methods are usually restricted to the second-order accuracy form, as the higher orders mean much higher complexity of the schemes.

One of the most frequently used channel flow DNS databases with heat transfer obtained with finite volume schemes has been maintained since 1998 by Kawamura. His team performed simulations of turbulent channel flow at various friction Reynolds numbers up to $\operatorname{Re}_{\tau}=1000$ and Prandtl numbers between $\operatorname{Pr}=0.025$ and 10 .

The recent DNS study of Vreman and Kuerten ${ }^{3}$ shows a detailed comparison of DNS results obtained with spectral and finite difference schemes. They found that their finite difference code (second-order spatial accuracy in periodic directions, fourth order in wall-normal direction, second order in time) required 3/4 smaller grid spacing than their spectral code to achieve the same accuracy.

Subgrid-scale LES models in finite volume/difference schemes are especially sensitive to numerical accuracy. The minimum requirement for LES simulations are second-order accurate schemes, although even higherorder schemes are sensitive to numerical dissipation when the grid resolution is used as a subgrid-scale filter width. From the mathematical point of view, the physical subgridscale models in finite difference methods are mainly introduced as additional eddy-viscosity terms with a space- and time-dependent viscosity. Various models of LES eddy viscosity models are today available in many commercial codes and also in the most popular open source codes like OpenFOAM and Code_Saturne. ${ }^{27,28}$ These codes are based on finite volume schemes with the maximum formal second-order accuracy. Thus, the use of these LES models, with a combination of physical and numerical dissipation of small scales, is recommended together with the use of best practice guidelines for CFD (Ref. 29).

\section{II.D. Other Numerical Schemes}

A number of other approaches have also been applied to DNS, like the lattice Boltzmann method, which has been shown to be capable of DNS and is known to be extremely efficient on massive parallel computers with graphics processing units due to its simplicity. ${ }^{30}$ Nevertheless, the method is not being widely used.

A relevant method, also in nuclear thermal hydraulics, is the spectral element method introduced in the 1980s. The method employs finite elements to discretize the domain and high-order Chebyshev or Legendre polynomials as the basis functions within each element. It has gained significant attention as a DNS tool thanks 
to the open-source code Nek5000 developed by Argonne National Laboratory ${ }^{31}$ (ANL). It is considered to be of similar accuracy as spectral schemes, with the advantage of being applicable to rather complex geometries and to be very efficiently parallelized on $10^{3}$ to $10^{4}$ CPUs. Besides the DNS capabilities, the spectral methods are used also for LES studies where the filtering of the small-scale eddies is performed on the level of each element in a similar way as in the spectral schemes.

\section{II.E. Boundary and Initial Conditions}

The specification of boundary conditions comes after the geometry, subgrid-scale model in LES, numerical scheme, and numerical resolution are selected for a DNS/LES run. The simplest boundary conditions applicable in the homogeneous direction are periodic boundary conditions. They are applicable also for the variables, which can be decomposed into the known mean gradient and homogeneous fluctuating part. Examples of such variables are pressure in the pipe or channel flow and temperature in the flow through the uniformly heated channel or pipe. In the case of spatially developing flows, one has to specify realistic turbulent inflow boundary condition. Various methods for generation of the inflow boundary conditions were recently reviewed by $\mathrm{Wu}^{32}$ They can be roughly divided into two approaches: a short auxiliary periodic domain is computed in parallel to the main calculation and is used to generate time-dependent inflow, or as an alternative, some of the faster but possibly less accurate "synthetic" methods can be used. The modeling of the outflow boundary condition is also not trivial. A review of the methods given in Ref. 33 shows that a certain part of the computational domain near the outlet surface gets contaminated by the outflow boundary condition.

Most of the DNS and LES computations are focused on statistically steady-state flows. These are flows with well-defined time-averaged statistics like mean velocities, root-mean-square fluctuations, etc. The statistical properties of DNS/LES simulations are discussed in Sec. III, while here only a few words about the initial conditions are needed. The initial conditions of the simulation can be selected as a sum of approximate mean velocity fields, which is close to the expected mean velocities, plus a random part of fluctuating velocities. If we are "unlucky," the fluctuations might die out and the flow laminarizes, but in most cases the flow will gradually develop into a "real" turbulent flow.

\section{INTERPRETATION OF DNS/LES RESULTS}

Depending on the detailed flow geometry, the statistically steady-state turbulence in DNS and LES is usually achieved after a time interval that typically spans over several flow-through times (from half a dozen and up to several dozen time units in channel flow and time normalized with $h / u_{\tau}$ ). The assessment of statistical steady state is all but trivial. In principle, every flow parameter - velocity, pressure or temperature at a given point, average value in a given line, on a selected surface or in a selected volume - should exhibit constant mean values with fluctuations around the means. In the turbulent channel flow, typical parameters used to identify statistical steady state are usually global variables like mean velocity, mean temperature, turbulence kinetic energy, friction velocities, and friction temperatures computed at the walls, etc. Typically, statistical independence must be observed over several dozens of flow-through times and over a dozen or several dozen time units in order to recognize it.

Instantaneous fields produced by DNS/LES, like the one shown in Fig. 1, are mainly used to produce nice pictures; however, they offer a rather limited amount of information. The accuracy of the instantaneous field cannot be verified as it cannot be directly compared to the experiments; this field is only one of the many possible realizations of the turbulent flow in a domain. Even if the calculation is correct and accurate, the problem of comparison with other calculations and experiments remain. Consistent comparison is possible only with statistically averaged results.

Once the statistical steady state is recognized, the temporal and, if possible, spatial averaging of the results can start. Typical averaging procedures include computations of mean values in a point, over the line, surface, or in the selected volume. Flow statistics can be computed on the fly during the simulation or a posteriori by using snapshots of the field variables. Both approaches have obvious advantages and drawbacks. Since the number of statistical quantities to be computed can reach an order of a 100 parameters (when various budget terms are evaluated), the choice of the postprocessing approach is influenced by the available computational and storage resources.

It might take very long computations to get DNS/LES results with sufficiently low statistical uncertainty. Figure 2 shows some examples for the channel flow (flow between 


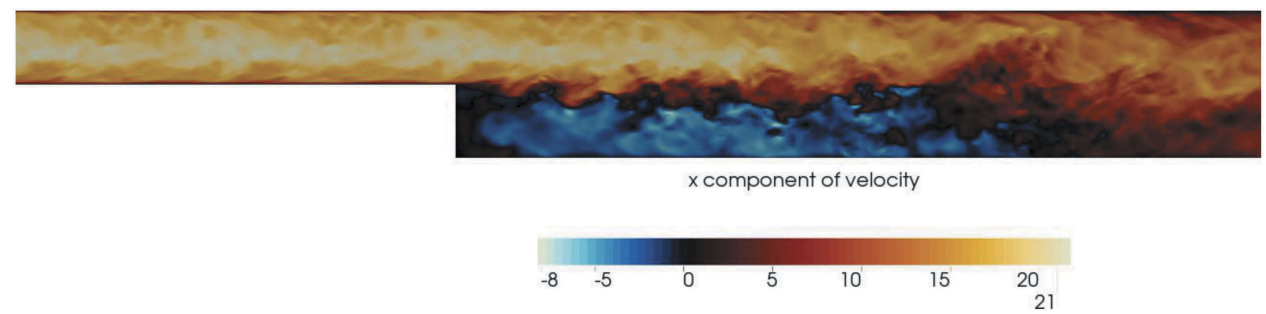

Fig. 1. Colorful velocity field of DNS simulations. Flow is from left to right.
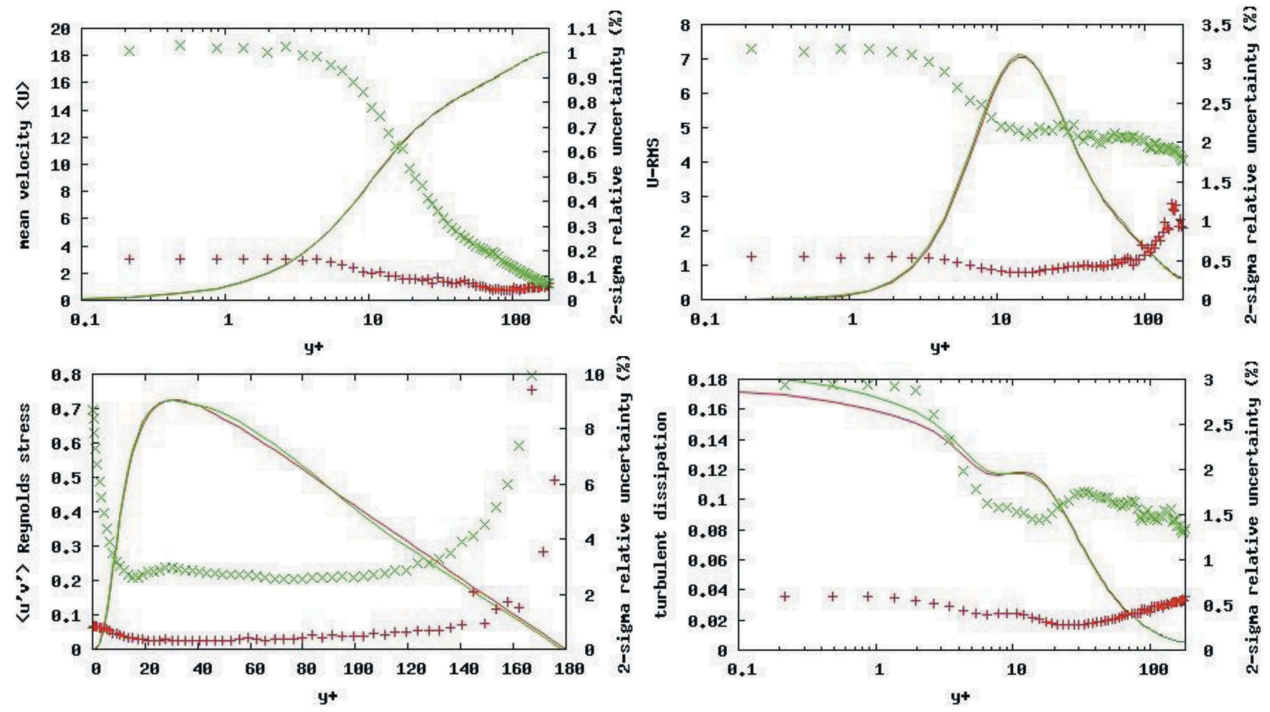

Fig. 2. Various turbulence statistics in channel flow with uncertainties (symbols): red represents spatial and temporal averaging and green represents temporal averaging only.

two infinite parallel walls), which is a geometry with two homogeneous directions (wall at $y=0$ ). The number of time steps needed for the acceptable statistical uncertainty might be rather large. A rather generally accepted rule of thumb says that the relative accuracy of the basic statistical profiles in DNS should be less than $\sim 1 \%$. However, a detailed study by Vreman and Kuerten ${ }^{3}$ shows that it takes several hundred time units (several hundred thousand time steps) to achieve that target even in channel flow with two homogeneous directions. The corresponding averaging time for the flows with a single homogeneous direction ${ }^{35}$ is longer and must be between one and two orders of magnitude longer in the geometries without homogeneous directions, where the only available type of averaging is averaging in time or ensemble averaging with several independent DNS runs performed in the same geometry.

A methodology and a tool for quantitative analyses of uncertainties in DNS (and LES) computations has been proposed in Ref. 36. Their tool is being used in this work to quantify the statistical uncertainty in channel flow heat transfer DNS. Figure 2 shows profiles of various turbulent statistics with quantified statistical uncertainty. Two types of averaging are used: much lower uncertainty is achieved when temporal averaging is combined with the spatial averaging in two homogeneous directions (red curves and symbols). Uncertainties are much larger when only temporal averaging is used in the same run (green curves and symbols). While not shown in the Fig. 2, we obtained the same statistical uncertainties in wall-resolved LES computations performed on the same computational domain and over the same time interval. Channel flow DNS in Fig. 2 was performed with the spectral method, second-order accurate in time, at $\operatorname{Re}_{\tau}=180$ in geometry and resolution of the (quasi)DNS in Ref. 20. The averaging time was 300 and the time step 0.00015 , both normalized with $h / u_{\tau}$ ( $h=$ channel half-width).

If the results shown in Fig. 2 are applicable for an arbitrary turbulence without homogeneous directions, then reduction of statistical uncertainty requires 
typically millions of time steps and consequently large computational power. An option for efficient analysis of such flows is running several statistically independent runs on a lower number of CPUs. This ensemble averaging approach might be computationally more efficient than running a single case on a very large number of CPUs and is also the only option for unsteady DNS and LES analyses.

Another question is related to homogeneous directions and periodic boundary conditions in DNS and LES: the question of the domain size in each homogeneous direction. Typical criteria, which ensure sufficient domain size, are two-point correlation functions. ${ }^{4}$ If the autocorrelation functions of each physical quantity of the flow computed in the homogeneous direction drop to zero at sufficient distance from the boundary, then the computational domain is considered as sufficiently long to describe all important large-scale structures of the flow. Figure $3 \mathrm{a}$, shows the streamwise autocorrelation functions of three components of velocity in DNS simulation from Fig. 2.

Figure $3 \mathrm{~b}$ shows the Fourier transformation of the streamwise velocity autocorrelation function, i.e., spectra of the streamwise velocity fluctuations. The spectra with resolution of the first DNS studies ${ }^{4}$ reveals a small nonphysical pile-up of the high frequency (quasi-DNS). The other spectra are obtained with roughly 7 times larger number of the Fourier modes [fully resolved DNS (Ref. 3)].

Despite the autocorrelation function criteria, there is no mechanism to accurately predict the scale of the largest structures in a given turbulent flow. As shown (among others) in our study, ${ }^{37}$ this question remains open even for the simple case of a channel flow. We have demonstrated the existence of very long turbulent structures, which have negligible impact on the flow field. However, their footprints remain visible in the low Prandtl number scalar field. Low Prandtl number fluid efficiently dissipates the small-scale turbulent structures and emphasizes the very large structures, which might be relevant for certain liquid metal applications.

\section{DNS IN NUCLEAR THERMAL HYDRAULICS: SOME EXAMPLES}

The channel flow DNS, with some characteristic results already given in Sec. III, is used for rather theoretical studies. Nevertheless, in some cases it can come very close to nuclear applications, especially in heat and mass transfer studies. One such case is our conjugate heat transfer DNS (Ref. 19) that took into account the detailed heat transfer in the solid heated walls of the channel and was performed for variable combinations of the low Prandtl number liquid (liquid metal) and solid material properties. Figure 4 shows the key parameter: penetration of the temperature turbulent fluctuations generated in the fluid into the solid wall heated by a constant density heat source.

A simple upgrade of the channel DNSs in the sense of geometry is the BFS. This geometry is a representative geometry for sudden expansions of pipes, channels, and ducts. Probably the first DNS of flow in a BFS geometry at a turbulent Reynolds number was performed in 1997 (Ref. 38), while the most recent DNS of heat transfer in this geometry can be found in Ref. 10 where the buoyant flow pattern in the BFS geometry with the expansion ratio 2 at Reynolds number of 10000 (based on step height and bulk velocity at inlet) and Prandtl number of sodium, $\operatorname{Pr}=0.0088$, was analyzed with one (spanwise) homogeneous dimension. Our research is focused on thermal fluctuations in the BFS geometry with solid walls. However, unlike previous simulations, our domain is closer to the experimental setup and does not contain a homogeneous direction. This prohibits the use of spatial averaging that is employed in DNS of infinitely wide BFS. The step wall and the lower wall behind the step are solid. The latter has a constant volumetric heat source.
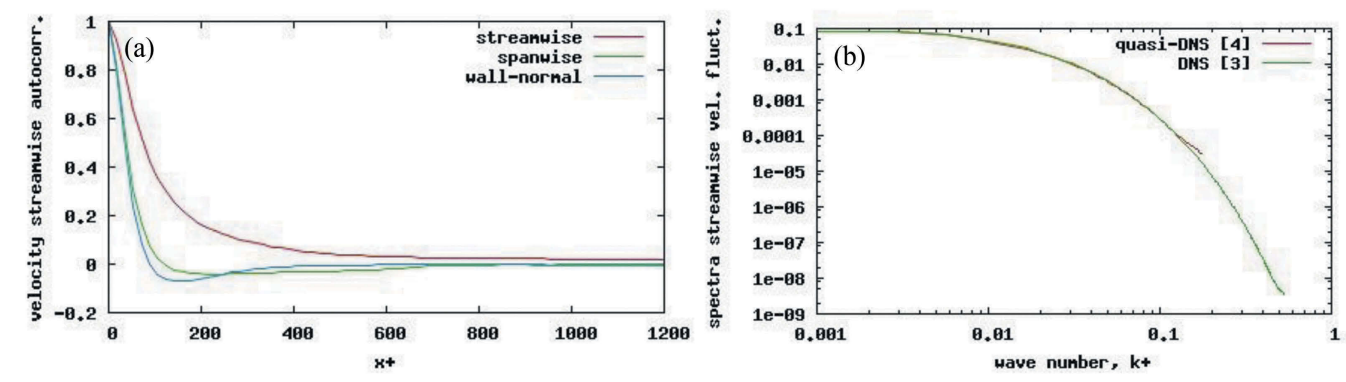

Fig. 3. (a) Streamwise autocorrelation functions of velocity components and (b) spectra of streamwise velocity fluctuations with quasi-DNS (Ref. 4) and DNS (Ref. 3) resolution. All at $y^{+}=180$. 


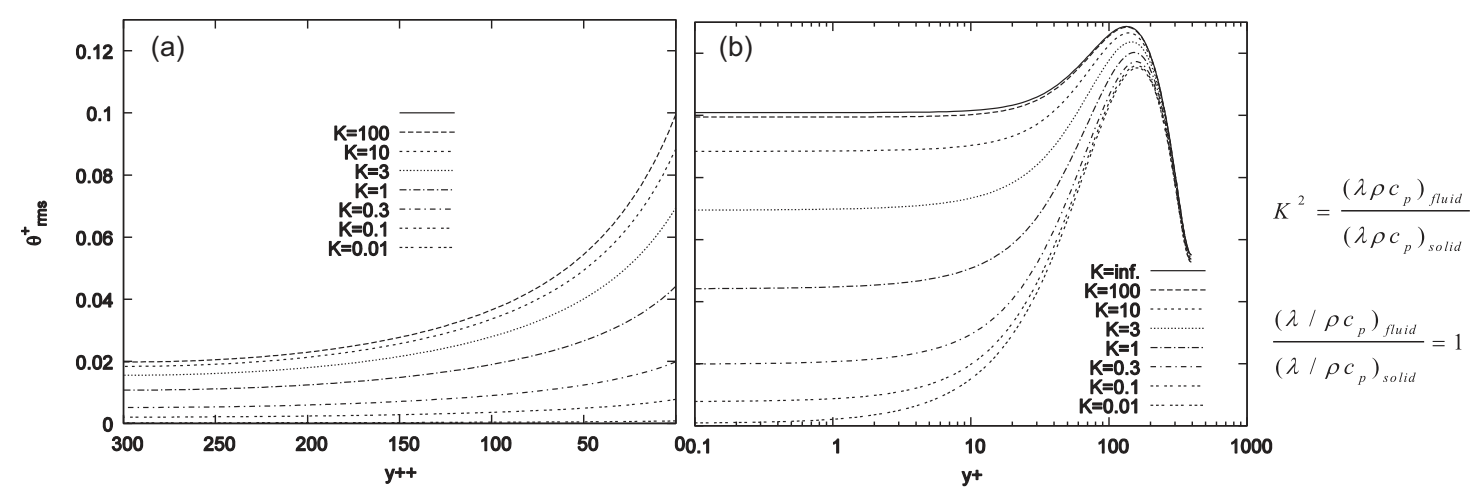

Fig. 4. (a) Wall and (b) fluid temperature fluctuations in $\operatorname{Pr}=0.01$ conjugate heat transfer channel flow DNS for variable material properties: $\operatorname{Re}_{\tau}=395$.

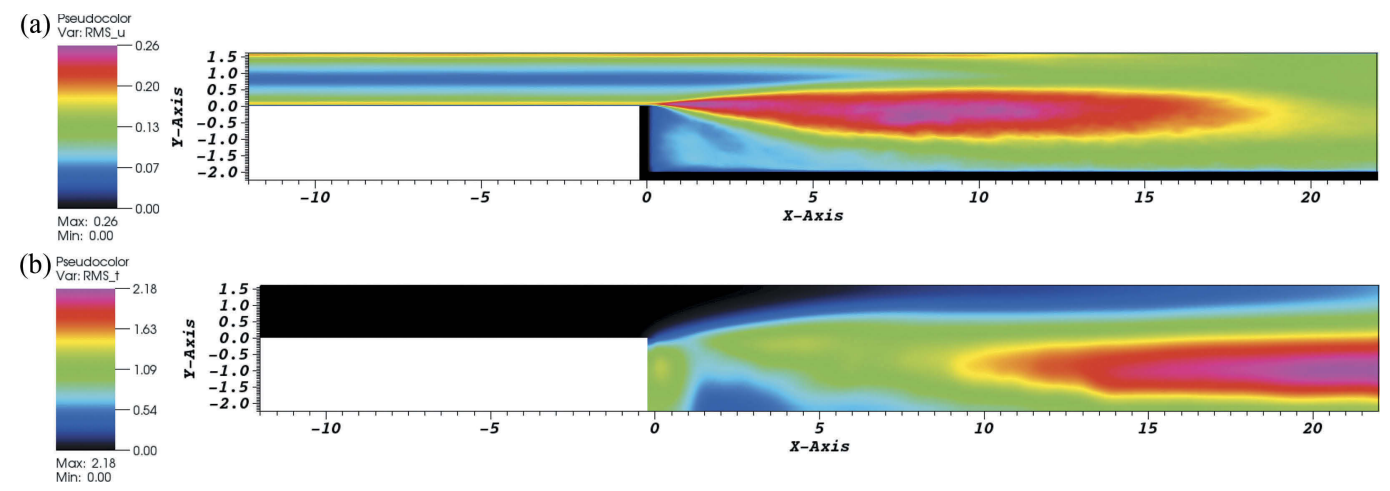

Fig. 5. (a) Streamwise velocity fluctuations and (b) temperature fluctuations in BFS DNS.

Figure 5 shows an example of the velocity fluctuation and temperature fluctuation fields in the BFS geometry.

Recently, the first DNS of turbulent heat transfer in a T-junction was performed. ${ }^{39}$ A larger horizontal channel with hot liquid joins with a vertical channel with colder liquid from above. The Reynolds number (based on the bulk velocity and channel half-width) in the horizontal channel was very low at 3000 , while the Reynolds number in the vertical channel was 343. The Prandtl number was equal to 1.44 . Buoyancy effects were taken into account with a parabolic relation between density and temperature. The effects of temperature-dependent viscosity and thermal diffusivity were also accounted for. The computational grid in this finite volume simulation consisted of around $5 \times 10^{7}$ nodes. The comparison with LES simulation of the same setup revealed that LES produced mildly diffused profiles for the secondorder statistics in the regions of intense turbulence production, however, the discrepancies are minor compared with the computational savings of LES. The mesh for LES had an order of magnitude less computational nodes.

The DNS of an impinging jet into parallel disks was performed in 1998 (Ref. 40) in a cylindrical domain with a jet with a Reynolds number of 10000 . Turbulent heat transfer in plane impinging jet was analyzed in Ref. 41 .

Slowly exceeding simple academic geometries, DNS is reaching toward geometries that are more complex. The results of a DNS of flow in a triangular-shaped rod bundle subchannel were presented in 2006 (Ref. 42). Recently (Refs. 11 and 43) a DNS of a flow pattern in a face-centeredcubic unit of a pebble bed structure in the pebble bed hightemperature reactor was presented and is shown in Fig. 6.

In 2015, Shams et al. ${ }^{44}$ presented quasi-DNS results of an infinite wire wrap around the fuel assembly of a liquid metal fast reactor. The study was conducted in a way where it retains the realistic design parameters of the MYRRHA reactor (Fig. 7).

\section{WALL-RESOLVED LES IN NUCLEAR THERMAL HYDRAULICS: SOME EXAMPLES}

As previously mentioned, the transition from the quasi-DNS into an accurate wall-resolved LES is rather continuous. LES, with typically at least one order of 


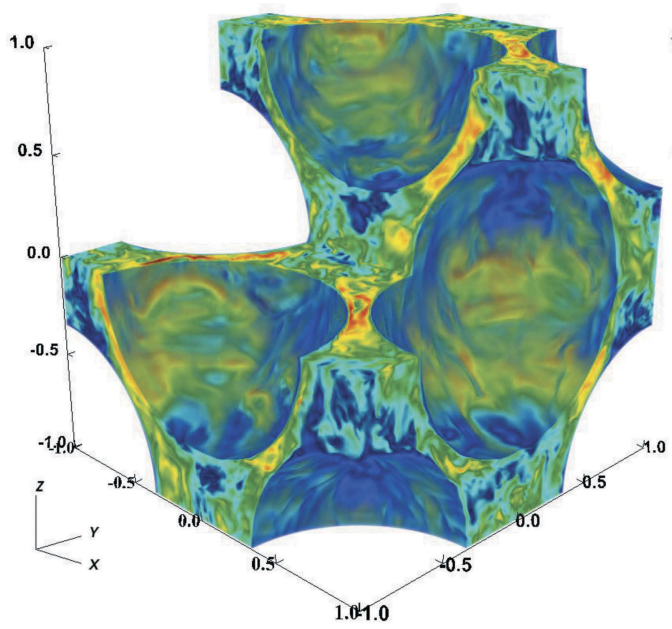

(a)

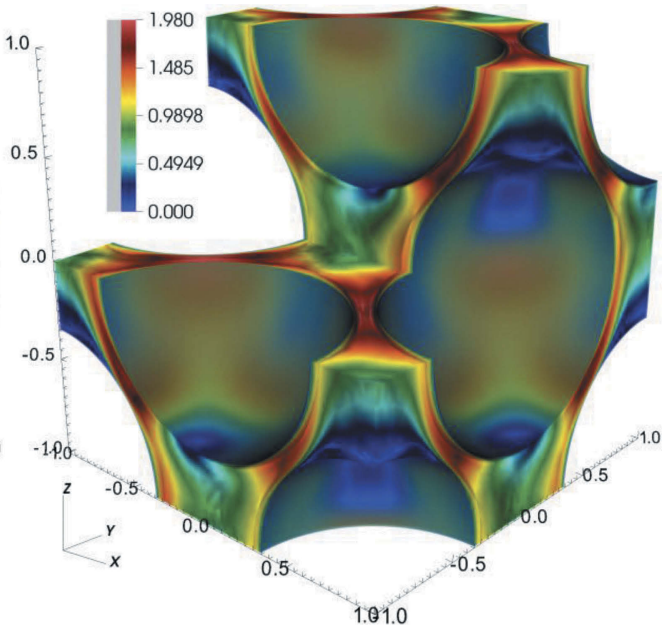

(b)

Fig. 6. (a) Instantaneous and (b) averaged velocity magnitude in the pebble bed (courtesy of ANL).

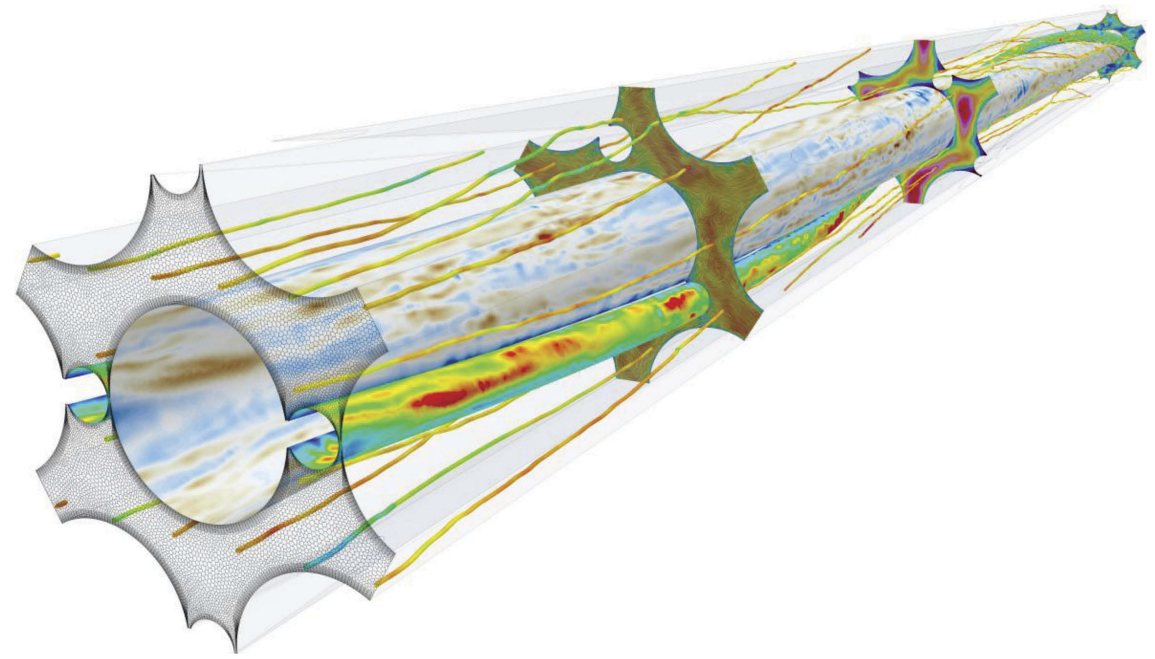

Fig. 7. Iso-surface of a criterion for identifying vortices (colors: velocity magnitude) from the quasi-DNS analysis of a wirewrapped fuel assembly (courtesy of NRG).

magnitude lower computational efforts, is thus a preferable method of choice for many nuclear applications. $^{45}$

Our recent research, where the use of LES was crucial, is thermal fatigue. Existing RANS models are not able to predict temperature fluctuations in a solid domain adjacent to a turbulent flow, ${ }^{46}$ and thus cannot investigate these issues. On top of that, there are doubts regarding the ability of wall-modeled LES to correctly predict the wall heat-flux spectrum in T-junctions, ${ }^{47}$ although it could predict the variance of the temperature in the wall. ${ }^{48}$ DNS and wall-resolved LES (Ref. 49) allowed us to create a database that can be used to develop new turbulence models able to tackle thermal fatigue issues.
In Ref. 50, Benhamadouche et al. showed that wallresolved LES can produce pressure loss and discharge coefficients in very good agreement with International Organization for Standardization standards regarding singlephase pipe flows through square-edged orifices. This enables one to study configurations without having to perform expensive experiments each time a new configuration is assessed. Again, it will help to improve industrial RANS models.

Our group was among several teams that have used wall-resolved LES to study the flow in a simplified fuel rod bundle. ${ }^{51}$ We have obtained mean values in very good agreement with experimental ones. Using a wall-resolved LES with a fine resolution, Walker et al. ${ }^{52}$ were able to accurately predict the local wall shear stress on a pin in 
a rod bundle. As shown by Yuan et al. ${ }^{53}$ such analyses allow the visualization of secondary flows and the measurement of flow-induced vibrations in SGs. Wallresolved LES was also used by Salkhordeh et al. ${ }^{54}$ to investigate the turbulent mixing in the lower plenum of a high-temperature gas-cooled reactor.

Although wall-modeled LES is out of the scope of the present work, the authors would like to point out a few single-phase applications of interest. One such remarkable result was the analysis of Bakosi et al. ${ }^{55}$ on turbulent statistics to study vibrations in fuel rod bundles. Other relevant applications include the work by Loginov et al. ${ }^{56}$ on the pressurized thermal shock (Fig. 8) and by Jayaraju et al. ${ }^{57}$ on boron dilution transient. According to Refs. 58 and 59, LES is also able to tackle containment issues, such as the erosion of a stratified layer by a buoyant jet.

\section{CONCLUSIONS AND CHALLENGES}

Following Bestion in Ref. 60, a large number of issues in nuclear thermal hydraulics can be simulated with CFD in the near future and most of the CFD studies of relevant industrial configurations will be performed with RANS, hybrid RANS-LES, or wallmodeled LES.

The DNS and wall-resolved LES methods, as the most accurate approaches, are computationally expensive and require large supercomputers. The aforementioned applications show that they are powerful and, especially LES, versatile tools able to tackle complex issues. And although the range of DNS and LES applications will grow, it is conjectured that wall-resolved LES, and of course DNS, of some full-scale industrial configurations will remain out of reach of supercomputers in the near future due to very large-scale separations.
Nevertheless, computationally very demanding wallresolved LES and DNS, alongside experiments, will remain the key to obtain accurate, detailed, and reproducible data in order to assess existing turbulence models, develop new ones, verify and validate CFD codes, and quantify uncertainty, thus improving the reliability of CFD for industrial applications and reactor safety assessments.

As shown above, wall-resolved LES remains the best candidate that will elucidate the details of the heat transfer in the cooling channels of existing and future reactors. It might become a key tool for the design of grid spacers and mixing vanes in light water reactor fuel, it might offer an insight into the fuel rod vibrations on the scale of the fuel pin. Pin-to-pin modeling of the whole reactor core will probably remain out of reach for wall-resolved LES in the next decade. However, heat and mass transfer problems related to mixing of emergency core cooling (ECC) system fluid with primary coolant in the pipes of the primary system, in the RPV downcomer, and at the bottom head of the RPV are problems that have already been tackled with LES in some recent works or will be approached in the near future. Perspectives of LES methods are foreseen also for multiphysics problems like conjugate heat transfer and more complicated fluid structure interactions.

Several relevant applications of (quasi)DNS and wall-resolved LES are available also in the field of GenIV reactors: in the geometries and with material properties of the liquid metal-cooled reactors, gascooled pebble bed or block reactors, or in the supercritical water reactors, where efficient and accurate modeling of buoyancy still represents a challenge for less accurate models. DNS, and especially wall-resolved LES, might significantly reduce the requirements for experiments needed for design and safety assessment of new reactors.

The available computational power has been continuously rising in the past decades and it is clear that this trend
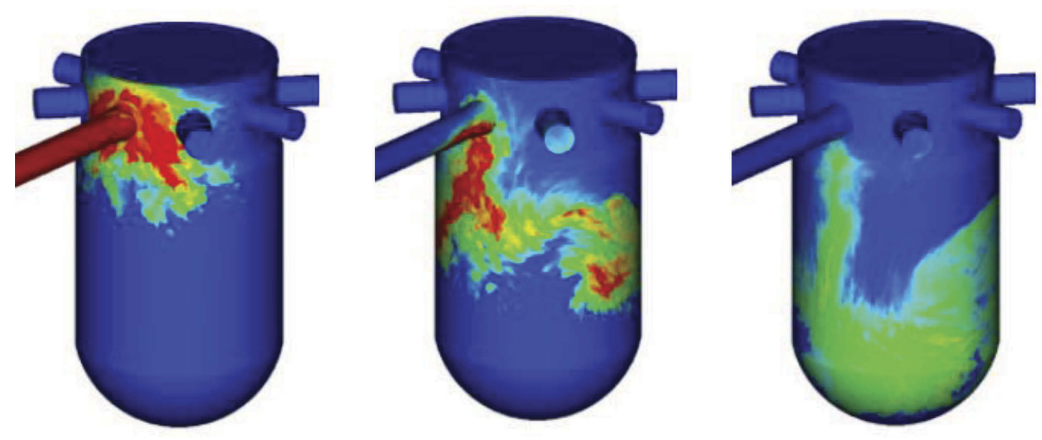

Fig. 8. Wall-modeled LES. Left: Visualization of ECC injection water temperature (red $=$ colder) during a Pressurized Thermal Shock transient (courtesy of NRG). 
will go on in the near future as machines in the exaflops range (a billion billion calculations per second) should be ready in 2020 to 2023 in China, the United States, Japan, and Europe. This paves the way for DNS and LES at higher Reynolds numbers and in more complex geometries that are closer to industrial needs. An important challenge for DNS/ LES techniques is permanent improvement of algorithms and computer codes in order to follow the development and to ensure efficient use of High Performance Computing resources. Numerical schemes for massively parallel computers might be significantly different from the traditional algorithms that were developed in the beginning of the CFD era. Even the algorithms that were abandoned as inefficient might become useful again, if they can be efficiently parallelized.

The increase of simulation size presents a challenge to the classical postprocessing strategy, thus promoting coprocessing that enables one to analyze and visualize data on the fly during the simulation. Thus, further interesting fields of research related to DNS and LES are methods of "big data" analyses, which are used to extract complex patterns from huge data sets. Another challenge related to data processing might be recognition and description of unsteady large-scale structures. Their presence might be important for many industrial systems. Today, most of the DNS and LES studies are focused on time-averaged results only.

The last recognized challenge, but not the least one, is that DNS and LES studies would benefit from the methods that would perform automatic analysis of statistical uncertainties of the predicted turbulent statistics.

\section{Acknowledgments}

This project has received funding from the Euratom research and training programme 2014-2018 under grant agreement number 654935 and from the Ministry of Higher Education, Science and Technology, Republic of Slovenia, Research programme P20026 and Research project L2-9210. Thanks to E. Merzari and L. Fick from ANL for their assistance with Fig. 6. Thanks to A. Shams and E. Komen from the Nuclear Research and consultancy Group (NRG) for their assistance with Figs. 7 and 8.

\section{ORCID}

Iztok Tiselj @ http://orcid.org/0000-0001-9340-5397

\section{References}

1. S. B. POPE, Turbulent Flows, Cambridge University Press, United Kingdom (2000).
2. C. D. ARGYROPOULOS and N. C. MARKATOS, "Recent Advances on the Numerical Modelling of Turbulent Flows," Appl. Math. Modell., 39, 2, 693 (2015); https://doi.org/10. 1016/j.apm.2014.07.001.

3. A. W. VREMAN and J. G. M. KUERTEN, "Comparison of Direct Numerical Simulation Databases of Turbulent Channel Flow at $\mathrm{Re}_{\tau}=180$," Phys. Fluids, 26, 015102 (2014); https://doi.org/10.1063/1.4861064.

4. J. KIM, P. MOIN, and R. MOSER, "Turbulence Statistics in Fully Developed Channel Flow at Low Reynolds Number,” J. Fluid Mech., 177, 133 (1987); https://doi.org/ 10.1017/S0022112087000892.

5. P. MOIN and K. MAHESH, "Direct Numerical Simulation: A Tool in Turbulence Research," Annu. Rev. Fluid Mech., 30, 539 (1998); https://doi.org/10.1146/annurev.fluid.30.1.539.

6. N. KASAGI, Y. TOMITA, and A. KURODA, "Direct Numerical Simulation of Passive Scalar Field in a Turbulent Channel Flow," ASME. J. Heat Transfer., 114, 3, 598 (1992); https://doi.org/10.1115/1.2911323.

7. I. TISELJ et al., "Effect of Wall Boundary Condition on Scalar Transfer in a Fully Developed Turbulent Flume Flow," Phys. Fluids, 13, 4, 1028 (2001); https://doi.org/ 10.1063/1.1350899.

8. I. TISELJ et al., "DNS of Turbulent Heat Transfer in Channel Flow with Heat Conduction in the Solid Wall," J. Heat Trans., 123, 849 (2001); https://doi.org/10.1115/1. 1389060.

9. I. OTIĆ, G. GRÖTZBACH, and M. WÖRNER, “Analysis and Modelling of the Temperature Variance Equation in Turbulent Natural Convection for Low-Prandtl-Number Fluids," J. Fluid Mech., 525 (2005); https://doi.org/10. 1017/S0022112004002733.

10. M. NIEMANN and J. FRÖHLICH, "Turbulence Budgets in Buoyancy-Affected Vertical Backward-Facing Step Flow at Low Prandtl Number," Flow Turbul. Combust., 99, 3-4, 705 (2017); https://doi.org/10.1007/s10494-017-9862-6.

11. L. H. FICK, E. MERZARI, and Y. A. HASSAN, "Direct Numerical Simulation of Pebble Bed Flows: Database Development and Investigation of Low-Frequency Temporal Instabilities," J. Fluids Eng., 139, 5 (2017); https://gfm.aps.org/meetings/dfd-2016/57da9f57b8a c311791000a2c (current as of Mar. 15, 2019).

12. M. LEE and R. D. MOSER, "Direct Numerical Simulation of Turbulent Channel Flow up to $\mathrm{Re}_{\tau}=5200$, , J. Fluid Mech., 774, 395 (2015); https://doi.org/10.1017/jfm.2015.268.

13. Y. YAMAMOTO and Y. TSUJI, "Numerical Evidence of Logarithmic Regions in Channel Flow at $\mathrm{Re}_{\tau}=8000$," Phys. Rev. Fluids, 3, 012602(R) (2018); https://doi.org/10. 1103/PhysRevFluids.3.012602.

14. P. SAGAUT, Large Eddy Simulation for Incompressible Flows: An Introduction, Springer Science \& Business Media (2006). 
15. C. MENEVEAU and J. KATZ, "Scale-Invariance and Turbulence Models for Large-Eddy Simulation," Annu. Rev. Fluid Mech., 32, 1 (2000); https://doi.org/10.1146/ annurev.fluid.32.1.1.

16. J. C. BOWMAN and M. ROBERTS, "Efficient Dealiased Convolutions Without Padding," SIAM J. Sci. Comput., 33, 1386 (2011); https://doi.org/10.1137/100787933.

17. S. HOYAS and J. JIMENEZ, "Scaling of the Velocity Fluctuations in Turbulent Channels up to Retau = 2003," Phys. Fluids, 18, 011702 (2006); https://doi.org/10.1063/1. 2162185.

18. J. GRAHAM et al., "A Web Services Accessible Database of Turbulent Channel Flow and Its Use for Testing a New Integral Wall Model for LES," J. Turbul., 17 (2016); https://doi.org/10.1080/14685248. 2015.1088656.

19. I. TISELJ and L. CIZELJ, "DNS of Turbulent Channel Flow with Conjugate Heat Transfer at Prandtl Number 0.01," Nucl. Eng. Des., 253, 153 (2012); https://doi.org/ 10.1016/j.nucengdes.2012.08.008.

20. D. MOSER, J. KIM, and N. MANSOUR, "Direct Numerical Simulation of Turbulent Channel Flow up to $\mathrm{Re}_{\tau}=$ 590," Phys. Fluids, 11, 4, 943 (1999); https://doi. org/10.1063/1.869966.

21. T. DAIRAY et al., "Numerical Dissipation Vs. Subgrid-Scale Modelling for Large Eddy Simulation," J. Comput. Phys., 337, 252 (2017); https://doi.org/10. 1016/j.jcp.2017.02.035.

22. F. F. GRINSTEIN, L. G. MARGOLIN, and W. J. RIDER, Implicit Large Eddy Simulation: Computing Turbulent Fluid Dynamics, Cambridge University Press (2007).

23. E. M. J. KOMEN et al., "A Quantification Method for Numerical Dissipation in Quasi-DNS and Under-Resolved DNS, and Effects of Numerical Dissipation in Quasi-DNS and Under-Resolved DNS of Turbulent Channel Flows," J. Comput. Phys., 345, 565 (2017); https://doi.org/10.1016/j. jcp.2017.05.030.

24. C. A. J. FLETCHER, Computational Techniques for Fluid Dynamics, 2nd ed., Springer, Berlin (1991).

25. J. D. ANDERSON, Computational Fluid Dynamics: The Basics with Applications, McGraw Hill (1995).

26. S. LAIZET, E. LAMBALLAIS, and J. VASSILICOS, "A Numerical Strategy to Combine High-Order Schemes, Complex Geometry and Parallel Computing for High Resolution DNS of Fractal Generated Turbulence," Comput. Fluids, 39, 3, 471 (2010); https://doi.org/10.1016/j.compfluid. 2009.09.018.

27. H. G. WELLER et al., "A Tensorial Approach to Computational Continuum Mechanics Using Object-Oriented Techniques," Comput. Phys., 12 (1998); https://doi.org/10.1063/1.168744.
28. F. ARCHAMBEAU, N. MÉCHITOUA, and M. SAKIZ, "Code_Saturne: A Finite Volume Code for the Computation of Turbulent Incompressible FlowsIndustrial Applications," Int. J. Finite, 1 (2004); http:// ijfv.math.cnrs.fr/IMG/pdf/saturne.pdf.

29. J. MAHAFFY et al., "Best Practice Guidelines for the Use of CFD in Nuclear Reactor Safety Applications," NEACSNI-R-2007-05, Organisation for Economic Cooperation and Development (2007); https://doi.org/10. 1094/PDIS-91-4-0467B.

30. G. MAYER and G. HAZI, "Direct Numerical and Large Eddy Simulation of Longitudinal Flow Along Triangular Array of Rods Using the Lattice Boltzmann Method," Math. Comput. Simul., 72, 173 (2006); https://doi.org/10. 1016/j.matcom.2006.05.011.

31. "Technical Report," Argonne National Laboratory(2017). https://nek5000.mcs.anl.gov/ (accessed Jan. 2017).

32. X. WU, "Inflow Turbulence Generation Methods," Annu. Rev. Fluid Mech., 49, 23 (2017); https://doi.org/10.1146/ annurev-fluid-010816-060322.

33. T. HATTORI et al., "Comparison of Non-Reflective Boundary Conditions for a Free-Rising Turbulent Axisymmetric Plume," Int. J. Numer. Methods Fluids, 72, 1307 (2013); https://doi.org/10.1002/fld.v72.12.

34. J. ODER, J. URANKAR, and I. TISELJ, "Spectral Element Direct Numerical Simulation of Heat Transfer in Turbulent Channel Sodium Flow," Proc. 24th Int. Conf. Nuclear Energy for New Europe (NENE2015), Portorož, Slovenia, September 14-17, 2015 (2015).

35. M. DUPONCHEEL and Y. BARTOSIEWICZ, "Low Prandtl Turbulent Heat Transfer in Unconfined or Wall-Bounded Configurations: DNS of a Mixing Layer and of an Impinging Jet," NURETH 17, Xi'an, China, September 4-9, 2017.

36. T. OLIVER et al., "Estimating Uncertainties in Statistics Computed from Direct Numerical Simulation," Phys. Fluids, 26, 3, 035101 (2014); https://doi.org/10.1063/1. 4866813.

37. I. TISELJ, "Tracking of Large-Scale Structures in Turbulent Channel with DNS of Low Prandtl Number Passive Scalar," Phys. Fluids, 26, 12, 125111 (2014); https://doi.org/10.1063/1.4905018.

38. H. LE, P. MOIN, and J. KIM, "Direct Numerical Simulation of Turbulent Flow over a Backward-Facing Step," J. Fluid Mech., 330, 349 (1997); https://doi.org/10. 1017/S0022112096003941.

39. M. GEORGIOU and M. V. PAPALEXANDRIS, "Direct Numerical Simulation of Turbulent Heat Transfer in a T-Junction," J. Fluid Mech., 845, 581 (2018); https://doi. org/10.1017/jfm.2018.256. 
40. S. SATAKE and T. KUNGI, "Direct Numerical Simulation of an Impinging Jet into Parallel Disks," Int. J. Numer. Methods Heat Fluid Flow, 8, 7, 768 (1998); https://doi. org/10.1108/09615539810232871.

41. H. HATTORI and Y. NAGANO, "Direct Numerical Simulation of Turbulent Heat Transfer in Plane Impinging Jet," Int. J. Heat Fluid Flow, 25, 5, 749 (2004); https://doi. org/10.1016/j.ijheatfluidflow.2004.05.004.

42. E. BAGLIETTO, H. NINOKATA, and T. MISAWA, "CFD and DNS Methodologies Development for Fuel Bundle Simulations," Nucl. Eng. Des., 236, 1503 (2006); https:// doi.org/10.1016/j.nucengdes.2006.03.045.

43. A. SHAMS et al., "Quasi-Direct Numerical Simulation of a Pebble Bed Configuration. Part I: Flow (Velocity) Field Analysis," Nucl. Eng. Des., 263, 473 (2013); https://doi. org/10.1016/j.nucengdes.2012.06.016.

44. A. SHAMS et al., "High Fidelity Numerical Simulations of an Infinite Wire-Wrapped Fuel Assembly," Nucl. Eng. Des., 335, 441 (2018); https://doi.org/10.1016/j. nucengdes.2018.06.012.

45. E. MERZARI et al., "Large-Scale Large Eddy Simulation of Nuclear Reactor Flows: Issues and Perspectives," Nucl. Eng. Des., 312, 86 (2017); https://doi.org/10.1016/j. nucengdes.2016.09.028.

46. C. FLAGEUL et al., "On the Discontinuity of the Dissipation Rate Associated with the Temperature Variance at the Fluid-Solid Interface for Cases with Conjugate Heat Transfer," Int. J. Heat Mass Transfer, 111, 321 (2017); https://doi.org/10.1016/j.ijheatmasstrans fer.2017.04.005.

47. S. T. JAYARAJU, E. M. J. KOMEN, and E. BAGLIETTO, "Suitability of Wall-Functions in Large Eddy Simulation for Thermal Fatigue in a T Junction," Nucl. Eng. Des., 240, 2544 (2010); https://doi.org/10.1016/j.nucengdes.2010.05.026.

48. J. A. HOWARD and É. SERRE, "Large Eddy Simulation in Code_Saturne of Thermal Mixing in a T Junction with Brass Walls," Int. J. Heat Fluid Flow, 63, 119 (2017); https://doi.org/10.1016/j.ijheatfluidflow.2016.09.011.

49. C. FLAGEUL, I. TISELJ, S. BENHAMADOUCHE, and M. FERRAND, "A Correlation for the Discontinuity of the Temperature Variance Dissipation Rate at the Fluid-Solid Interface in Turbulent Channel Flows," Flow Turbul. Combust., 103, 1, 175 (2019); https://doi.org/10.1007/ s10494-019-00008-0.

50. S. BENHAMADOUCHE, M. ARENAS, and W. J. MALOUF, "Wall-Resolved Large Eddy Simulation of a Flow Through a
Square-Edged Orifice in a Round Pipe at $\mathrm{Re}=25,000$," $\mathrm{Nucl}$. Eng. Des., 312, 128 (2017); https://doi.org/10.1016/j. nucengdes.2016.09.010.

51. B. MIKUŽ and I. TISELJ, "Wall-Resolved Large Eddy Simulation in Grid-Free $5 \times 5$ Rod Bundle of MATiS-H Experiment," Nucl. Eng. Des., 293, 64 (2016); https://doi. org/10.1016/j.nucengdes.2015.12.021.

52. J. WALKER et al., "Accurate Prediction of the Wall Shear Stress in Rod Bundles with the Spectral Element Method at High Reynolds Numbers," Int. J. Heat Fluid Flow, 50, 287 (2014); https://doi.org/10.1016/j.ijheatfluidflow.2014.08. 012.

53. H. YUAN et al., "Flow-Induced Vibration of a Helical Coil Steam Generator Experiment Using Large Eddy Simulation," Nucl. Eng. Des., 322, 547 (2017); https://doi. org/10.1016/j.nucengdes.2017.07.029.

54. S. SALKHORDEH et al., "Large Eddy Simulations of Scaled HTGR Lower Plenum for Assessment of Turbulent Mixing," Nucl. Eng. Des., 334, 24 (Aug. 2018); https://doi. org/10.1016/j.nucengdes.2018.04.016.

55. J. BAKOSI et al., "Large-Eddy Simulations of Turbulent Flow for Grid-to-Rod Fretting in Nuclear Reactors," Nucl. Eng. Des., 262, 544 (2013); https://doi.org/10.1016/j. nucengdes.2013.06.007.

56. M. S. LOGINOV, E. M. J. KOMEN, and A. K. KUCZAJ, "Application of Large-Eddy Simulation to Pressurized Thermal Shock: Assessment of the Accuracy," Nucl. Eng. Des., 241, 3097 (2011); https://doi.org/10.1016/j. nucengdes.2011.05.027.

57. S. T. JAYARAJU et al., "Large Eddy Simulation for an Inherent Boron Dilution Transient," Nucl. Eng. Des., 262, 484 (2013); https://doi.org/10.1016/j.nucengdes.2013.05. 024.

58. F. S. SARIKURT and Y. A. HASSAN, "Large Eddy Simulations of Erosion of a Stratified Layer by a Buoyant Jet," Int. J. Heat Mass Transfer, 112, 354 (2017); https:// doi.org/10.1016/j.ijheatmasstransfer.2017.04.134.

59. A. KRAUS et al., "Erosion of Large-Scale Gaseous Stratified Layer by a Turbulent Jet-Simulations with URANS and LES Approaches," 16th Int. Topl. Mtg, Nuclear Reactor Thermal Hydraulics (NURETH-16), Chicago, Illinois, August 30-September 4, 2015 (2015).

60. D. BESTION, "BEPU Methods Using CFD CodesProgress Made Within OECD-WGAMA CFD Activities," Proc. ANS Best Estimate Plus Uncertainties Int. Conf., Luca, Italy, May 13-18, 2018 (2018). 